\title{
Patterns of care in psychiatric case-register areas
}

\author{
ROBERT GIEL and SJOERD SYTEMA
}

During the past few decades totally independent psychiatric institutions have been replaced by or developed into systems or networks of services delivering mental health care. This was in line with WHO's guidelines of proper care, stipulating the need for continuity, flexibility and integration of care according to the changing needs of the patients.

Nowadays, it is accepted that patients are client of more than one institution, sometimes during one and the same episode of illness. This has complicated the follow up of individual careers in psychiatric care, which can no longer be studied by considering just one component of the network. An additional problem is that we can no longer trust mental hospitals of other institutions to reflect together the total administrative morbidity in an area. It is possible that in a census the same patient is counted twice or more, thereby inflating prevalence rates.

The varying need of psychiatric patients and the often chronic nature of their disorder have raised interest in the pattern of care they receive over a long period of time. This requires the coupling of care data per individual patient, not only of their contacts with a single component in the service network, but also with different components. Such linking of data is the specific aim of a case register. The general assumption is that patterns of care are somehow related to outcome, and that it is worthwile to study their efficiency and effectiveness for the various diagnostic categories of patients.

Indirizzo per la corrispondenza: Prof. R. Giel, Department of Social Psychiatry, University of Groningen, Postbox 30.001, 9700 RB Groningen, The Netherlands.

Fax: (+31) 050-696727.

\section{BUILDING PATTERNS OF CARE}

Basic to the description of a pattern of care is the definition of its 'building stones' i.e. the contact and the episode. Commonly, three types of contacts are distinguished: face-to-face contacts in an extramural or outpatient setting; day or partial care contacts in a semimural setting, various forms of sheltered housing are usually included here; and admission days in residential care (e.g. mental hospital).

Defining the episode is more arbitrary. For example, as an uninterrupted series of contacts within the period of observation. Of course, what constitutes an interruption has to be determined. This can be the formal termination of treatment, or an agreed minimum number of days between two subsequent contacts. In the latter case it is arbitrarily decided that, for example, contact after an interruption of at least three months without any contact sets off a new episode of illness. A related issue is the minimum duration or sequence of contacts an episode should have, in order to qualify as such. One can set a criterion of a minimum number of contacts of a similar nature (days in hospital, series of outpatient contacts or days in day care) for a care episode to qualify as such. This series of contacts should take place within a specified period of time. For example, an admission should last at least 24 hours; day care should count at least three contacts within a span of three week-days; outpatient care should count at least three contacts within 90 days. This is sometimes referred to as a step in an episode of care (Tricot, 1986, Giel et al., 1987).

So far, it will be clear that our a priori definition of the building stones of a pattern will strongly influence its shape. 


\section{EPISODES OF ILLNESS AND OF CARE}

Research into patterns of care requires a longitudinal approach, which is exactly what a case register offers as the data base of all contacts from a circumscribed population with all mental health services providing care to that population.

The period of observation for researching patterns of care has to be the same for each patient. Obviously, the natural course of the illness should ideally determine the lenght of the period of observation. At the same time, it has to be remembered that an episode of care and an episode of illness as determined by its symptoms need not always coincide. General health care or a long symptomatic period without any care may precede contact with the mental health services. Similarly, the end of the period of observation puts an artificial end to both the episode of care and that of illness.

\section{ClASSIFICATION OF PATTERNS OF CARE}

If research into patterns of care is to make sense, they have somehow to be ordered or even hierarchically ranked. Four dimensions are commonly used to arrive at a classification: the type of care, its setting, and the intensity and duration of care. The combination of these dimensions demands that choices be made.

A first and simple way of, for example, comparing register areas is by the distribution of cases on census day over the types of care: in-, semi- and extramural care. Next come the episodes of care experienced by a cohort of patients in any of these types, separately or combined, during the period of observation.

Giel and ten Horn (1982) attempted to include intensity (frequency of contacts) and duration in their classification: less than one outpatient contact per two weeks over a period of less than three months; one or more contacts per two weeks over a period of less than three months; the same variation of the frequency of contacts, this time during three months or more; and one or more admissions, with no more than one outpatient contact, or with more than one of such contacts. The period of observation is a limiting factor in this classification. Besides, the relationship between episodes of illness and episodes of care remains intractable. Finally, what exactly happens after three months is not taken into account.

Since such a classification is largely nominal in nature, ranking of patterns along a dimension of, for example, intensity is not really possible. This is regrettable, because such ranking would help us to assess the effectiveness of particular patterns of care.

Weighting the different types of care against each other in order to arrive at an ordinal or interval scale became the next concern. It required a criterion of intensity. Lavik (1983) proposed a cumulative measure by accrediting 1 point to one outpatient contact, 2 points to one day care contact, and 3 points to one day in hospital. He justified the weights by taking into account the time devoted to care and by its content. After adding all weighted contacts the «index of the total consumption of care» is obtained.

Thus defined, patients admitted during the full period of observation become the heaviest consumers of care. This does not strike one as a particularly adequate reflection of the intensity of the care rendered to a longstay patient as compared with an outpatient receiving frequent psychotherapy during only part of the period of observation.

Tansella et al. (1986) tried to get closer to letting the complexity of the patterns of care induce their classification, by proposing a statistical criterion for dividing the service consumption index into proper classes of heavy and non-heavy use. This implied searching for the score for which the ratio intergroup/intragroup variance is at its maximum. This score is used as the cut-off point for distinguishing heavy from non-heavy consumption of care. In other words, the two classes are chosen in such a way that they differ to the maximum degree possible, while internally each is as homogeneous as possible. $\mathrm{Pa}$ tients with one outpatient contac only and those permanently admitted are treated as separate classes.

After having identified the heavy and non-heavy users, the dimension of duration can be added at will. This whole procedure is dominated by the heavy weight of a day in hospital, while the pattern of utilization of the various types of care is obscured.

Kontny et al. (1982) and ten Horn and Haffner (1988) proposed arbitrary hierarchical classifications of care utilization, which are really no more than elaborations of the attempts of Giel and Ten Horn (1982) and Lavik (1983). In each case hospital care is assigned the highest level in the hierarchy, higher than any number of outpatient contacts.

The last of such a priori and arbitrary ways of arriving at a classification of patterns of care was 
introduced by Giel et al. (1987) and Sytema et al. (1989). However, like Tansella's classification it is somewhere in between the a priori classifications discussed above and the more dynamic ones to be mentioned next, in that the duration of care in addition to the intensity (frequency) of contacts is statistically accounted for. The following measures are used, yielding interval scales of care utilization:

Gross duration of care $=$ number of days between first and last contact in the period of observation.

Net duration of care $=$ gross duration minus number of days in a period of no care. A period of no care is defined according to the step criterion of minimally 90 days without any contact.

Intensity of gross care $=$ the relation between net and gross care, i.e. the proportion of the gross care period spent in net care.

Admission ratio = the total numer of admissions divided by the net duration of care.

These measures are summary statistics denoting a patient's career in mental health care during the period of observation.

\section{DYNAMIC ANALYSIS OF PATTERNS OF CARE}

Service utilization, nowadays, is a dynamic process. It is likely that a number of transitions will take place in a patient's career. For example, from out- to inpatient care, or from outpatient to out-of-care, etc.

In the traditional approaches, described above, a dynamic process is forced to fit a static classificatory model. The introduction and development of dynamic methods for the analysis of longitudinal data (Event History Analysis) allowed researchers to model dynamic processes in a more dynamic way (Blossfeld et al., 1989; Tuma \& Hannan, 1984).

There are at least three reasons for doing so:

1. Traditional methods are dependent on the time frame chosen arbitrarily in advance. For example, in traditional studies about the risk of readmission, a percentage will be calculated relating to the duration of the follow-up period. In survival analysis the variations in the risk during the follow-up period are the object of study, which is much more informative than a single number statistic. Moreover, when the risk of readmission is not constant over time, traditional studies using different follow-up periods will result in different conclusions. This is prevented by using survival methods.
2. In the readmission example, patients not readmitted before the end of the follow-up period cause a problem in traditional calculations, because their risk of readmission remains unknown. Researchers usually collapse the data into two groups: readmitted yes/no. However, patients readmitted within the follow-up period may differ from those readmitted outside it. Dichotomization conceals such differences. With survival methods, analysis of the risk of readmission over time, such differences will be demonstrated. Data on patients without a readmission will be treated as censored, i.e. all data obtained will be used up to the end of the follow-up period showing for which number of patients the risk is as yet undecided.

3. In a dynamic process the effect of the predictors may vary over time. For example, aftercare contacts may delay readmission, but later on the risk of readmission may equal that of patients without such contacts. Variations over time in the effect of predictors will show up in survival analysis. Moreover, time-dependent covariates (for example, the time, until the aftercare contact) can be introduced into the analysis.

Two functions representing processes of change will probably appear to be most useful in studying patterns of care:

1. The transition probability function. This function represents the chance of going from one state (e.g. inpatient care) to another (e.g. outpatient care or out-of-care) within fixed time intervals.

2. The hazard function. This function represents the chance of an event occurring within specified time intervals in the category of the cohort at risk at the start of such a time interval.

A simple application of both functions was shown by Häfner and an der Heiden (1983) in a study of a cohort of schizophrenic patients. They distinguished between three states of care (inpatient, extramural aftercare and no aftercare). At $\mathrm{t} 1$ all patients were in hospital. In each of 36 consecutive intervals of two weeks the distribution of the cohort over the three states was observed. It was found that after one year a stable distribution over the states was reached $(17 \%$ hospitalized; $20 \%$ no care; $63 \%$ aftercare). A firstorder Markov model could adequately describe the process of transitions between the states. In such a model the distribution over the states at $t(i)$ depends totally on their distribution at $t(i-1)$. The whole process can be described with one transition probability matrix.

They used a simple survival model to describe the 
risk of readmission after discharge for a group with extensive and another with medium utilization of complementary services.

Although complex dynamic models appear promising for studying patterns of care, traditional a priori classifications remain useful because of their intuitive appeal and for planning purposes. Dynamic analysis can be used to test assumptions implicit in traditional classifications or to construct new classifications.

\section{CONCLUSION}

At this stage, critical readers will be inclined to ask why they should bother with all this complex business of patterns of care (Hansson \& Sondlund, 1992). We think that they serve to describe and compare service networks, which should be the concern of mental health planners and managers. In order to evaluate a service you have to know what it is doing. Next, by altering components of introducing new ones, and studying their effect on patterns of care, the latter can be improved.

Mental health workers will be interested in the illness careers of various categories of patients: similar groups of people with different diseases or different groups of people (e.g. the sexes) with the same disease. They will want to be able to predict those careers, to search for effective interventions. Therefore, they will be interested in the relationships between patterns of care and predictive characteristics of their patients, and between patterns of care and outcome.

\section{REFERENCES}

Blossfeld H. P., Hamerle A. \& Mayer K. U. (1989). Event Histo$r y$ Analysis. Statistical Theory and Application in the Social Sciences. Lawrence Erlbaum Associates: New Jersey.

Giel R. \& ten Horn G. H. M. M. (1982). Patterns of mental health care in a Dutch register area. Sccial Psychiatry 19, 117-123.

Giel R., Hermann P., ten Horn G. H. M. M. \& Tricot L. (1987). Patterns of care and service utilization. In Mental Health Services in Study Pilot Areas (ed. R. Giel, J. H. Hannibal, J. H. Henderson and G. H. M. M. ten Horn), pp. 77-117. WHO Regional Office for Europe: Copenhagen.

Giel R., Sytema S., Brook F. G. \& ten Horn G. H. M. M. (1987), Patterns of care of depressive illness: a case-register study towards a hierarchical classification. In Psychiatric Epidemiology: Progress and Prospects (ed. B. Cooper), pp. 247-256. Croom Helm: London/Sydney.

Häfner H. \& an der Heiden W. (1983). The impact of a changing system care on patterns of utilization by schizophrenics. Social Psychiatry 18, 153-160.

Hansson L. \& Sandlund M. (1992). Utilization and patterns of care in comprehensive psychiatric organizations. Acta Psychiatrica Scandinavica 86, 255-261.

ten Horn G. H. M. M. \& Haffner J. (1988). The types of treatment and care psychiatric patients receive: an attempt to standardize different measures and patterns of care. Unpublished.

Kontny E. L., Cooper J. E. \& Howat J. G. M. (1982). The patient who is new to psychiatric services. Nottingham. Unpublished.

Lavik N. J. (1983). Utilization of mental health services over a given period. Acta Psychiatrica Scandinavica 67, 404-413.

Sytema S., Giel R. \& ten Horn G. H. M. M. (1989). Patterns of care in the field of mental health. Acta Psychiatrica Scandinavica $79,1-10$.

Tansella M., Micciolo R., Balestrieri M. \& Gavioli I. (1986). High and long-term users of the mental health services. Social Psychiatry 21, 96-103.

Tricot L. (1986). An example of the standardization of terminology: defining episodes of care. In Psychiatric Case Registers in Public Health (ed. G. H. M. M. ten Horn, R. Giel, W. H. Gulbinat and J. H. Henderson). Elsevier: Amsterdam.

Tuma N. B. \& Hannan M. T. (1984). Social Dynamics: Models and Methods. Academic Press: New York. 